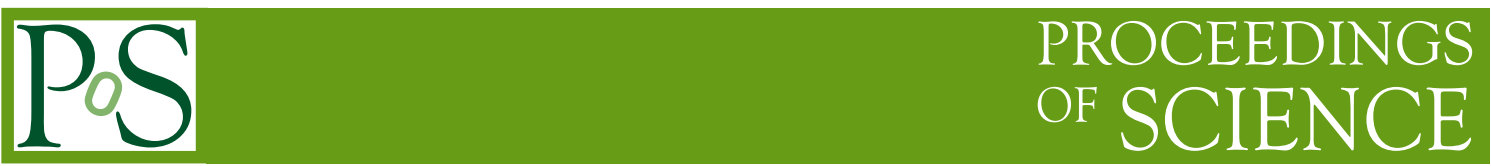

\title{
Science with the Australian SKA pathfinder
}

\author{
Simon Johnston* ${ }^{* \dagger}$ \\ Australia Telescope National Facility, CSIRO \\ E-mail: Simon. Johnston@csiro.au
}

\begin{abstract}
The future of $\mathrm{cm}$ and $\mathrm{m}$-wave astronomy lies with the Square Kilometre Array (SKA), a telescope under development by a consortium of 17 countries that will be 50 times more sensitive than any existing radio facility. Most of the key science for the SKA will be addressed through large-area imaging of the Universe at frequencies from a few hundred $\mathrm{MHz}$ to a few $\mathrm{GHz}$. The Australian SKA Pathfinder (ASKAP) is a technology demonstrator aimed in the mid-frequency range, and achieves instantaneous wide-area imaging through the development and deployment of phased-array feed systems on parabolic reflectors. The large field-of-view makes ASKAP an unprecedented synoptic telescope that will make substantial advances in SKA key science. ASKAP will be located at the Murchison Radio Observatory in inland Western Australia, one of the most radio-quiet locations on the Earth and one of two sites selected by the international community as a potential location for the SKA. In this paper, we outline an ambitious science program for ASKAP, examining key science such as understanding the evolution, formation and population of galaxies including our own, understanding the magnetic Universe, revealing the transient radio sky and searching for gravitational waves.
\end{abstract}

From planets to dark energy: the modern radio universe October 1-5 2007

University of Manchester, Manchester, UK

\footnotetext{
*Speaker.

${ }^{\dagger}$ ASKAP Project Scientist
} 


\section{Introduction}

The Australian SKA Pathfinder (ASKAP) is a next generation radio telescope on the strategic pathway towards the staged development of the Square Kilometre Array (SKA). The ASKAP project is international in scope and includes partners in Australia, Canada, the Netherlands and South Africa. In this short conference paper I give an overview of the ASKAP project and give a brief description of the planned science program. An expanded write-up of the science case for ASKAP can be found in the refereed paper [1]. Details and updates for the ASKAP program generally (including positions vacant!) can be found on the web at wwwatnf.atnf.csiro.au/projects/askap/

ASKAP has three main goals:

- to carry out world-class, ground breaking observations directly relevant to the SKA Key Science Projects;

- to demonstrate and prototype technologies for the mid-frequency SKA, including field-ofview enhancement by focal-plane phased-arrays on new-technology 12-m class parabolic reflectors;

- to establish a site for radio astronomy in Western Australia where observations can be carried out free from the harmful effects of radio interference.

\section{ASKAP System Parameters}

Table 1 gives the ASKAP system parameters. The first column gives the relevant parameter with the second column listing the base model parameters for ASKAP. The stretch goals are given in the third column; implementation of these depend on the addition of further dishes and/or the cooling of the focal plane array elements to provide a lower system temperature.

\subsection{Survey Speeds}

There are two important metrics for telescopes like ASKAP. The first is the survey speed; the amount of sky which can be surveyed in a given time to a given sensitivity. The second is the instantaneous sensitivity. The first metric can be expressed as a proportionality $\propto A^{2} T^{-2} F$, where $A$ is the total (effective) collecting area $\left(\mathrm{m}^{2}\right), T$ the system temperature $(\mathrm{K})$ and $F$ the field of view (sq deg). Instantaneous sensitivity is proportional to $A T^{-1}$. The first two entries of Table 2 lists these values for ASKAP. The survey speed for ASKAP is 1\% of that expected from the full SKA in this frequency band.

In the remaining rows of Table 2 we list values of the sensitivity and survey speed for different 'typical' surveys for both the base model and and the stretch parameters. The third entry gives a continuum survey where the entire $300 \mathrm{MHz}$ of bandwidth is exploited and a desired 1- $\sigma$ sensitivity of $100 \mu \mathrm{Jy}$ is required. The fourth entry gives a spectral line survey, with the fifth line listing a surface brightness survey needing to reach a $1-\sigma$ limit of $1 \mathrm{~K}$ over $5 \mathrm{kHz}$ channel under the assumption of a $1^{\prime}$ resolution. The final row lists the time necessary to reach 1- $\sigma$ of 1 mJy across a $1 \mathrm{MHz}$ bandwidth to a point source at the centre of the field. The stretch goals for ASKAP offers a factor of almost 5 improvement in survey speed over the base model design. 
Table 1: System parameters for ASKAP

\begin{tabular}{lcc}
\hline \multicolumn{1}{c}{ Parameter } & Base Parameters & Stretch Goals \\
\hline Number of Dishes & 30 & 45 \\
Dish Diameter $(\mathrm{m})$ & 12 & 12 \\
Dish area $\left(\mathrm{m}^{2}\right)$ & 113 & 113 \\
Total collecting area $\left(\mathrm{m}^{2}\right)$ & 3393 & 5089 \\
Aperture Efficiency & 0.8 & 0.8 \\
System Temperature $(\mathrm{K})$ & 50 & 35 \\
Number of beams & 30 & 30 \\
Field-of-view (deg $\left.{ }^{2}\right)$ & 30 & 30 \\
Frequency range $(\mathrm{MHz})$ & $700-1800$ & $700-1800$ \\
Instantaneous Bandwidth (MHz) & 300 & 300 \\
Maximum number of channels & 16000 & 16000 \\
Configuration maximum baseline (m) & 2000 & 400,8000 \\
\hline
\end{tabular}

\subsection{Configuration}

A number of science projects (pulsar surveys, Galactic H I, low surface brightness mapping) require a highly compact array configuration in order to increase the surface brightness survey speed. On the other hand science such as continuum and transients require long baselines both to overcome the effects of confusion and to obtain accurate positions for identification at other wavelengths. In the middle is the extragalactic $\mathrm{H}$ I survey which needs moderate resolution to avoid over-resolving the sources. With a total of only 30 dishes it is difficult to achieve all these requirements simultaneously. The base parameters therefore include a single configuration with a maximum baseline of $\sim 2 \mathrm{~km}$, ideal for the $\mathrm{H}$ I survey and good for the transient and polarization surveys.

For the stretch goals, it is envisaged that two further array configurations will be available, a very compact configuration (maximum baseline $\lesssim 400 \mathrm{~m}$ ) and an extended configuration (maximum baseline $\sim 8 \mathrm{~km}$ ). Configuration changes would occur by physically moving the antennas and would happen only infrequently.

A design study is currently underway to determine exact antenna positions for different configurations given the science case and the constraints of the local site topology and terrain.

\subsection{Location}

The central core of ASKAP will be located at the Murchison Radio Observatory in inland Western Australia, one of the most radio-quiet locations on the Earth and one of the sites selected by the international community as a potential location for the SKA. The approximate geographical coordinates of the site are longitude 116.5 east and latitude 26.7 south. The southern latitude of ASKAP implies that the Galactic Centre will transit overhead and the Magellanic Clouds will be prominent objects of study. At least 30,000 square degrees of sky will be visible to ASKAP. The choice of site ensures that ASKAP will be largely free of the harmful effects of radio interference 
Table 2: Sensitivity and survey speeds for ASKAP

\begin{tabular}{|c|c|c|c|}
\hline Parameter & Base model & Stretch goals & \\
\hline Survey speed & $8.8 \times 10^{4}$ & $4.1 \times 10^{5}$ & $\mathrm{~m}^{4} \mathrm{~K}^{-2} \mathrm{sq} \operatorname{deg}$ \\
\hline Instantaneous sensitivity & 54 & 116 & $\mathrm{~m}^{2} \mathrm{~K}^{-1}$ \\
\hline Continuum survey speed (300 MHz, $100 \mu \mathrm{Jy})$ & 250 & 1150 & $\mathrm{deg}^{2} / \mathrm{hr}$ \\
\hline Line survey speed $(100 \mathrm{kHz}, 5 \mathrm{mJy})$ & 209 & 960 & $\mathrm{deg}^{2} / \mathrm{hr}$ \\
\hline Surface brightness survey speed $\left(5 \mathrm{kHz}, 1 \mathrm{~K}, 1^{\prime}\right)$ & 18 & 83 & $\mathrm{deg}^{2} / \mathrm{hr}$ \\
\hline Point source sensitivity ( $1 \mathrm{MHz}, 1 \mathrm{mJy})$ & 1290 & 280 & $\mathrm{sec}$ \\
\hline
\end{tabular}

currently plaguing the present generation of telescopes, especially at frequencies around $1 \mathrm{GHz}$ and below. Being able to obtain a high continuous bandwidth at low frequencies is critical to much of the science planned for ASKAP.

\subsection{Timeline}

In early 2008, the ASKAP test bed antenna will be installed at the site of the 64-m Parkes radio telescope to allow testing of the focal plane array and beamforming systems. Following this, the goal is to have the first 6 antennas of ASKAP on-site in Western Australia in early 2010. Over the subsequent two years the remainder of the antennas would be deployed, with commissioning of the final system expected to take place in late 2012.

\section{Science with ASKAP}

Following international science meetings held in Australia in April 2005 and March 2007, seven main science themes have been identified for ASKAP. These are extragalactic H I science, continuum science, polarization science, Galactic and Magellanic science, VLBI science, pulsar science and the radio transient sky. In this paper, I can do no more than list bullet points for each science section. An extended version of the science case has already been published [1], with larger, and more complete, version of the science case for ASKAP still to be published.

The technological innovation of ASKAP and the unique radio quiet location in Western Australia will enable a powerful synoptic survey instrument that will make substantial advances in SKA technologies and on three of the SKA key science projects: the origin and evolution of cosmic magnetism, the evolution of galaxies and large scale structure, and strong field tests of gravity. The headline science goals for ASKAP are:

- The detection of a million galaxies in atomic hydrogen emission across $80 \%$ of the sky out to a redshift of 0.2 to understand galaxy formation and gas evolution in the nearby Universe (see Figure 1).

- The detection of synchrotron radiation from 60 million galaxies to determine the evolution, formation and population of galaxies across cosmic time and enabling key cosmological tests. 


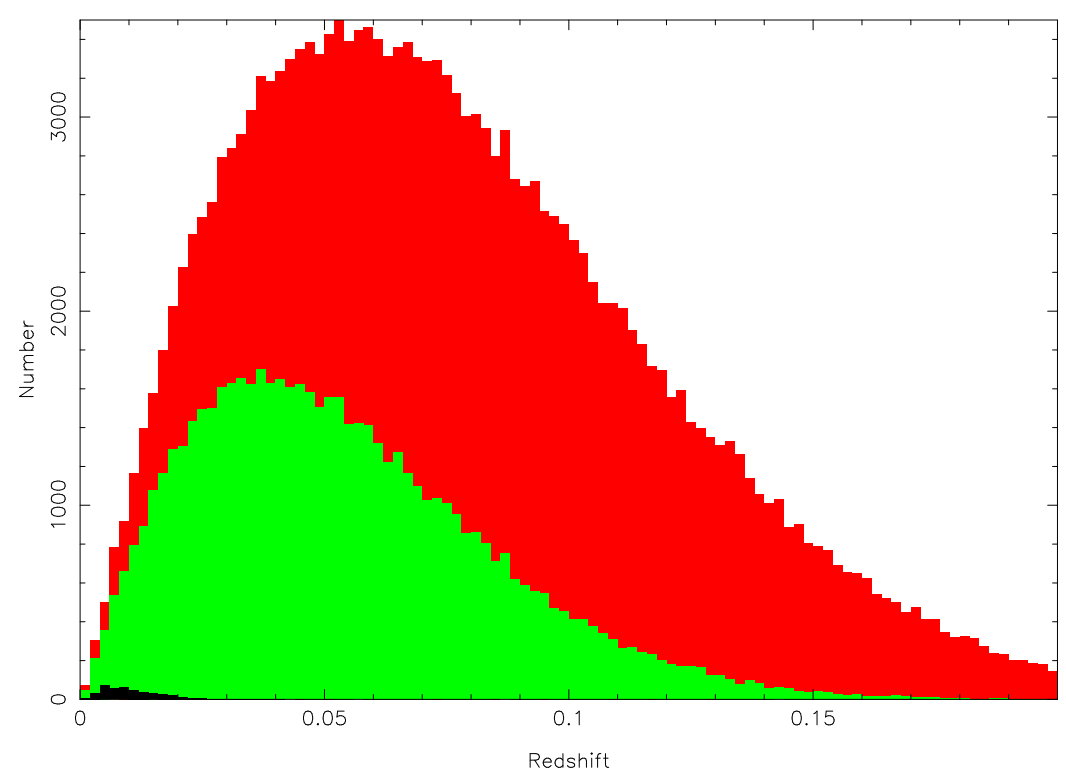

Figure 1: Number of galaxies above $5 \sigma$ as a function of redshift bin for a shallow ASKAP H I survey lasting a year, covering the southern hemisphere, compared with HIPASS HICAT (ref [2]; black histogram). The simulation assumes the an HIMF with no evolution [3], and a bivariate relation between H I mass and velocity width. The number of predicted detections is $\sim 1.8 \times 10^{6}$ and $\sim 0.6 \times 10^{6}$ for the stretch (red histogram) and base model (green histogram) options.

- The detection of polarized radiation from over 500,000 galaxies, allowing a grid of rotation measures at $10^{\prime}$ to explore the evolution of magnetic fields in galaxies over cosmic time.

- The understanding of the evolution of the interstellar medium of our own Galaxy and the processes that drive its chemical and physical evolution.

- The characterization of the radio transient sky through detection and monitoring of transient sources such as gamma ray bursts, radio supernovae and intra-day variables.

- The discovery and timing of up to 1000 new radio pulsars to find exotic objects and to pursue the direct detection of gravitational waves.

- The high-resolution imaging of intense, energetic phenomena through improvements in the Australian and global Very Long Baseline networks.

\section{References}

[1] S. Johnston et al., 2007, PASA, In Press (http://xxx.lanl.gov/abs/0711.2103)

[2] M. Meyer et al., 2004, MNRAS, 350, 1195

[3] M. Zwaan et al., 2005, MNRAS, 359, 30 